Nota de investigación

\title{
Densidades de plantas y dosis de fertilización en el cultivo de amaranto
}

\author{
Carlos Osvaldo Romero Romano ${ }^{1 \S}$ \\ Juventino Ocampo Mendoza ${ }^{1}$ \\ Engelberto Sandoval Castro ${ }^{1}$ \\ Hermilio Navarro Garza ${ }^{2}$ \\ Omar Franco Mora ${ }^{3}$
}

${ }^{1}$ Colegio de Postgraduados-Campus Puebla. Boulevard Forjadores de Puebla núm. 205, Santiago Momoxpan, San Pedro Cholula, Puebla, México. CP. 72760. Tel. 222 2850738. (jocampo@colpos.mx; engelber@colpos.mx). ${ }^{2}$ Colegio de Postgraduados-Campus Montecillo. Carretera México-Texcoco km 36.5, Texcoco, Estado de México, México. CP. 56230. Tel. 595 9520200. (hermnava@colpos.mx). ${ }^{3}$ Universidad Autónoma del Estado de México-Campus El Cerrito. Carretera Toluca-Ixtlahuaca, Piedras Blancas km 15.5, Toluca de Lerdo, Estado de México, México. CP. 50200. Tel. 7222965554. (ofrancom@uaemex.mx).

${ }^{\S}$ Autor para correspondencia: carlos.romero@ colpos.mx.

\section{Resumen}

El objetivo de esta investigación fue evaluar la respuesta de Amaranthus hypochondriacus L. raza Mercado a la densidad de plantas y dosis de fertilización en las variables: rendimiento, altura de planta, diámetro de tallo, tamaño de panoja, e índice de plantas acamadas. Los factores de estudio fueron: densidad (D) de plantación (156 250, 208333 y 260417 plantas ha $^{-1}$ ), niveles de nitrógeno $\left(40,80\right.$ y $120 \mathrm{~kg} \mathrm{ha}^{-1}$ ), niveles de potasio (cero y $20 \mathrm{~kg} \mathrm{ha}^{-1}$ ) y aplicación de $40 \mathrm{~kg} \mathrm{ha}^{-1}$ de fósforo como una constante. El arreglo de tratamientos consistió en una factorial 3x3x2 en un diseño experimental de bloques completamente al azar con cuatro repeticiones y 18 tratamientos. El experimento se realizó en los ciclos de cultivo 2017 y 2018 en Tochimilco, Puebla, México. Las labores de cultivo se realizaron acorde al itinerario técnico del sistema convencional de siembra directa que consiste en colocar en cada hoyo de siembra un número variable de semillas para asegurar que en cada hoyo crezcan varias plantas y posteriormente realizar un aclareo, en el que se arrancan las plantas más pequeñas y se dejan tres, cuatro o cinco plantas en cada hoyo de siembra dependiendo de la experiencia de cada agricultor. Los resultados muestran diferencias altamente significativas $(p<0.01)$ en rendimiento, altura de planta, longitud y ancho de panoja por efectos individuales e interacciones de $\mathrm{D}, \mathrm{N} * \mathrm{~K}$ y $\mathrm{N} * \mathrm{~K} * \mathrm{D}$. Se concluyó, que la decisión que toman los productores de dejar tres, cuatro o cinco plantas por hoyo de siembra es una decisión determinante en la producción del amaranto.

Palabras clave: Amaranthus hypochondriacus L., itinerario técnico, sistema convencional de siembra directa.

Recibido: julio de 2021

Aceptado: agosto de 2021 
El amaranto (Amaranthus spp.) es reconocido a nivel internacional como un cultivo que puede contribuir a incrementar la seguridad alimentaria mundial y ser una alternativa para zonas que se ven afectas por los efectos adversos del cambio climático debido a sus propiedades nutracéuticas y sus cualidades agronómicas (Alemayehu et al., 2015). En México, Amaranthus hypochondriacus L., es una de las especies domesticadas por las culturas precolombinas; actualmente, se han identificado cuatro razas nativas de A. hypochondriacus (Azteca, Mercado, Mixteca y Nepal) las cuales presentan diferente distribución geográfica y características fenológicas y morfológicas (Espitia et al., 2020).

De acuerdo con la información presentada en el Sistema de Información Agroalimentaria y Pesquera (SIAP, 2017), en México se siembran 3190.95 ha de amaranto en seis entidades federativas, siendo Puebla el estado con mayor superficie sembrada (1 975.1 ha). Asimismo, al interior del estado, el municipio de Tochimilco es el principal productor con $1467.2 \mathrm{t}$ anuales, cantidad que equivale al $56.71 \%$ de la producción estatal y $29.2 \%$ de la producción nacional. No obstante, Tochimilco tiene uno de los rendimientos promedios más bajos del país $\left(1.31 \mathrm{t} \mathrm{ha}^{-1}\right)$, debido principalmente a la alta incidencia de plagas y enfermedades (Romero et al., 2018) y la aplicación de dosis de fertilización deficientes (Sánchez et al., 2016).

Aunado a estos factores, el rendimiento de amaranto también puede verse afectado por las características propias del sistema de producción utilizado, en el caso de Tochimilco el sistema que se practica es el convencional de siembra directa. En este sistema la siembra se efectúa por medio de la técnica de mateado la cual consiste en colocar en cada hoyo de siembra un número variable de semillas con la finalidad de asegurar que en cada hoyo germinen y crezcan varias plantas.

Posteriormente, 30 días después de la siembra (dds), se realiza un aclareo de plantas que consiste en arrancar las plantas más pequeñas y dejar tres, cuatro o cinco plantas en cada hoyo de siembra dependiendo de la lógica productiva de cada agricultor (Romero et al., 2017), de tal forma que, considerando el número de plantas por hoyo de siembra, la separación entre hoyos $(30 \mathrm{~cm})$, el ancho de los surcos $(40 \mathrm{~cm})$ y la separación entre cada surco $(60 \mathrm{~cm})$, la densidad de plantas puede ser 156 250, 208333 o 260417 plantas ha ${ }^{-1}$ respectivamente. Por tanto, el objetivo de esta investigación fue evaluar la respuesta de A. hypochondriacus raza Mercado a tres densidades de plantación y diferentes dosis de fertilización en las variables rendimiento de grano, altura de planta, diámetro de tallo, longitud y ancho de panoja, e índice de plantas acamadas.

\section{Localización}

La investigación se realizó durante los ciclos de cultivo primavera-verano de los años 2017 y 2018 en una parcela de un productor cooperante localizada en la comunidad Santa Catarina Tepanapa, perteneciente al municipio de Tochimilco, Puebla, en la región central de México (18 ${ }^{\circ} 50^{\prime}$ y $19^{\circ}$ $02^{\prime}$ latitud norte y los meridianos $97^{\circ} 18^{\prime}$ y $97^{\circ} 27^{\prime}$ de longitud oeste) a una altitud de $2280 \mathrm{~m}$, con clima templado subhúmedo con lluvias en verano C(w1) y precipitación anual de $1100 \mathrm{~mm}$. En febrero de 2017, se realizó un análisis del suelo de la parcela en el laboratorio de suelos del Colegio de Postgraduados Campus Montecillo, para conocer sus características físico-químicas más relevantes, los resultados se muestran en la Cuadro 1. 
Cuadro 1. Características físico-químicas del suelo de la parcela experimental localizada en Santa Catarina Tepanapa, Tochimilco, Puebla.

\begin{tabular}{cccccccc}
\hline $\mathrm{pH}$ & $\mathrm{CE}\left(\mathrm{dS} \mathrm{m}^{-1}\right)$ & $\mathrm{Nt}(\%)$ & $\mathrm{P}(\mathrm{ppm})$ & $\mathrm{K}\left(\mathrm{meq} 100 \mathrm{~g}^{-1}\right)$ & $\mathrm{CIC}\left(\mathrm{meq} 100 \mathrm{~g}^{-1}\right)$ & $\mathrm{MO}(\%)$ & Textura \\
\hline 7.85 & 0.468 & 0.081 & 5.78 & 0.68 & 7.76 & 1.45 & Franco arenosa \\
\hline
\end{tabular}

Datos del análisis de suelo realizado en el laboratorio del Colegio de Postgraduados, Campus Montecillo (2017). CE= conductividad eléctrica; $\mathrm{Nt}=$ nitrógeno total $; \mathrm{P}=$ fósforo; $\mathrm{K}=$ potasio $; \mathrm{CIC}=$ capacidad de intercambio catiónico; $\mathrm{MO}=$ materia orgánica.

\section{Diseño experimental y de tratamientos}

El arreglo de tratamientos consistió en un factorial $3 \times 3 \times 2$ correspondiente a tres densidades de plantación: 156 250, 208333 y 260417 plantas ha $^{-1}$ (equivalentes a dejar en cada hoyo de siembra tres, cuatro y cinco plantas respectivamente); tres dosis de nitrógeno: 40, 80 y $120 \mathrm{~kg} \mathrm{ha}^{-1}$; dos dosis de potasio: cero y $20 \mathrm{~kg} \mathrm{ha}^{-1}$ y $40 \mathrm{~kg} \mathrm{ha}^{-1}$ de fósforo como una constante. En total se evaluaron 18 tratamientos. Se utilizó un diseño experimental de bloques completamente al azar con cuatro repeticiones, la unidad experimental fue de tres surcos de $1.2 \mathrm{~m}$ de largo por $0.4 \mathrm{~m}$ de ancho, distancia entre surco $0.6 \mathrm{~m}\left(2.88 \mathrm{~m}^{2}\right)$, cada surco con cinco hoyos de siembra distanciados cada 0.3 m. Para evitar el efecto de bordo, se consideró como parcela útil el surco central.

\section{Labores de cultivo y cuantificación de variables}

En el experimento se sembró A. hypochondriacus raza Mercado el cual es uno de los materiales genéticos que cultivan los productores locales. Esta raza se caracteriza por tallos y hojas elípticas de color verde, semillas de color blanco o dorado, puede alcanzar hasta dos $\mathrm{m}$ de altura de planta, desarrolla numerosas ramificaciones laterales y tiene un ciclo biológico de 140 días y (Espitia et al., 2010). Las labores de cultivo se realizaron conforme al itinerario técnico del sistema convencional de siembra directa practicado por los productores locales. En el ciclo de cultivo 2017 la siembra se realizó el 30 de mayo en tanto que en el ciclo de cultivo 2018 la siembra se llevó el 3 de junio, una vez comenzado el ciclo anual de lluvia debido a que la producción es de secano.

En ambos ciclos, las dosis de fertilización se aplicaron en dos momentos. Los fertilizantes utilizados fueron urea (46-00-00), fosfato diamónico (18-46-00) y cloruro de potasio (00-00-60). La primera fertilización se realizó a los $10 \mathrm{dds}$, al momento del primer aporque, con $50 \%$ de la dosis de $\mathrm{N}$ y $100 \%$ de la dosis de $\mathrm{P}$ y K. La segunda fertilización con el otro $50 \%$ de la dosis de N se aplicó 60 dds, en el segundo aporque. Los deshierbes fueron manuales y antes de cada aporque. El aclareo de plantas se efectuó a los 30 dds, dejando tres, cuatro o cinco plantas por hoyo de siembre de acuerdo con cada uno de los tratamientos en estudio.

La última semana de octubre, cuando más de $90 \%$ de las plantas alcanzaron la madurez fisiológica, se midió en la parcela útil la altura de planta $(\mathrm{cm})$ desde la base del tallo hasta el extremo de la panoja, con un estadal Geosurv ${ }^{\circledR}$, el diámetro de tallo $(\mathrm{cm})$ a $10 \mathrm{~cm}$ sobre la base del tallo, con un vernier digital Surtek ${ }^{\circledR}$, la longitud $(\mathrm{cm})$ y ancho $(\mathrm{cm})$ de la panoja con un flexómetro metálico tipo 1-A, modelo: 334, ADIR, además se contó el número de plantas acamadas (plantas con inclinación de $45^{\circ}$ o más) y posteriormente en gabinete se calculó el índice de plantas acamadas (IPA), dividiendo el número de plantas acamadas entre el número total de plantas de la parcela útil, siendo el valor mínimo cero y el valor máximo uno. 
El corte de panoja se realizó en la segunda semana de noviembre, etapa en que $90 \%$ de las plantas alcanzaron su madurez, la cual se determina por la senescencia y caída de hojas. Las panojas se cortaron con una hoz y se colocaron sobre los surcos para que se secaran al sol. Un mes después del corte se efectuó la trilla del grano de forma manual, golpeando la panoja con varas para desprender las semillas sobre una manta. Los granos desprendidos se cernieron con un tamiz de acero inoxidable de $20 \mathrm{~cm}$ de diámetro con orificios de $16 \mathrm{~mm}$ para separar el tamo. Posteriormente se pesó la semilla en una balanza electrónica Medi data ${ }^{\circledR}$ de $500 \mathrm{~g}$, para cuantificar el rendimiento de grano $(\mathrm{g})$.

\section{Análisis estadístico}

Un análisis de varianza y una prueba de comparación de medias para efectos individuales y de interacción con base en la prueba de Tukey $(p<0.05)$ fueron realizados con el software Statistical Analysis System (SAS, 2004).

Las variables diámetro de tallo y el IPA no presentaron diferencias estadísticamente significativas. No obstante, se encontraron diferencias significativas $(p<0.05)$ en las variables rendimiento, altura de planta, longitud y ancho de panoja (Cuadro 2). El rendimiento máximo (1 $970.24 \mathrm{~kg} \mathrm{ha}^{-1}$ ) que se alcanzó con aplicaciones de $120 \mathrm{~kg} \mathrm{ha}^{-1}$ de $\mathrm{N}, 40 \mathrm{~kg} \mathrm{ha}^{-1}$ de P, $20 \mathrm{~kg} \mathrm{ha}^{-1}$ de K y densidad de 260417 plantas $\mathrm{ha}^{-1}$, superó 50.4\% al rendimiento promedio registrado por el SIAP (2017) en el municipio (1 $310 \mathrm{~kg} \mathrm{ha}^{-1}$ ). Estos resultados confirman lo propuesto por Sánchez et al. (2016) quienes mencionan que rendimientos cercanos a $2000 \mathrm{~kg} \mathrm{ha}^{-1}$ son alcanzables en las condiciones ambientales de Tochimilco con la adecuada nutrición del cultivo.

Cuadro 2. Valores medios de las variables evaluadas en dos ciclos de cultivo (2017 y 2018).

\begin{tabular}{ccccccc}
\hline $\begin{array}{c}\text { Tratamiento } \\
\text { N-P-K-D }\end{array}$ & $\begin{array}{c}\text { Rendimiento } \\
\left(\mathrm{kg} \mathrm{ha}^{-1}\right)\end{array}$ & $\begin{array}{c}\text { Altura de } \\
\text { planta }(\mathrm{m})\end{array}$ & $\begin{array}{c}\text { Diámetro de } \\
\text { tallo }(\mathrm{cm})\end{array}$ & $\begin{array}{c}\text { Longitud de } \\
\text { panoja }(\mathrm{cm})\end{array}$ & $\begin{array}{c}\text { Ancho de } \\
\text { panoja }(\mathrm{cm})\end{array}$ & IPA \\
\hline $40-40-0-3$ & $876.49 \mathrm{~d}$ & $1.2 \mathrm{ab}$ & $0.97 \mathrm{a}$ & $25.34 \mathrm{ab}$ & $5.99 \mathrm{ab}$ & $0.08 \mathrm{a}$ \\
$80-40-0-3$ & $924.11 \mathrm{~cd}$ & $1.18 \mathrm{ab}$ & $0.83 \mathrm{a}$ & $24.12 \mathrm{ab}$ & $5.34 \mathrm{ab}$ & $0 \mathrm{a}$ \\
$120-40-0-3$ & $1489.58 \mathrm{abcd}$ & $1.65 \mathrm{a}$ & $1.02 \mathrm{a}$ & $29.04 \mathrm{ab}$ & $8.51 \mathrm{a}$ & $0.17 \mathrm{a}$ \\
$40-40-20-3$ & $1452.38 \mathrm{abcd}$ & $1.3 \mathrm{ab}$ & $0.93 \mathrm{a}$ & $25.78 \mathrm{ab}$ & $6.43 \mathrm{ab}$ & $0.08 \mathrm{a}$ \\
$80-40-20-3$ & $1840.77 \mathrm{ab}$ & $1.42 \mathrm{ab}$ & $1.09 \mathrm{a}$ & $28.53 \mathrm{ab}$ & $8.01 \mathrm{ab}$ & $0.14 \mathrm{a}$ \\
$120-40-20-3$ & $944.94 \mathrm{bcd}$ & $1.16 \mathrm{ab}$ & $0.91 \mathrm{a}$ & $23.42 \mathrm{ab}$ & $6.46 \mathrm{ab}$ & $0 \mathrm{a}$ \\
$40-40-0-4$ & $958.33 \mathrm{bcd}$ & $1.25 \mathrm{ab}$ & $0.9 \mathrm{a}$ & $24.1 \mathrm{ab}$ & $6.23 \mathrm{ab}$ & $0.04 \mathrm{a}$ \\
$80-40-0-4$ & $910.71 \mathrm{~cd}$ & $1.13 \mathrm{ab}$ & $0.8 \mathrm{a}$ & $21.73 \mathrm{ab}$ & $5.95 \mathrm{ab}$ & $0.08 \mathrm{a}$ \\
$120-40-0-4$ & $1794.64 \mathrm{abc}$ & $1.5 \mathrm{ab}$ & $1 \mathrm{a}$ & $25.88 \mathrm{ab}$ & $7.07 \mathrm{ab}$ & $0.15 \mathrm{a}$ \\
$40-40-20-4$ & $1052.08 \mathrm{bcd}$ & $1.26 \mathrm{ab}$ & $0.93 \mathrm{a}$ & $27.22 \mathrm{ab}$ & $6.17 \mathrm{ab}$ & $0.06 \mathrm{a}$ \\
$80-40-20-4$ & $1610.12 \mathrm{abcd}$ & $1.22 \mathrm{ab}$ & $1.04 \mathrm{a}$ & $29.95 \mathrm{a}$ & $6.72 \mathrm{ab}$ & $0 \mathrm{a}$ \\
$120-40-20-4$ & $1126.49 \mathrm{abcd}$ & $1.29 \mathrm{ab}$ & $0.87 \mathrm{a}$ & $22.5 \mathrm{ab}$ & $5.53 \mathrm{ab}$ & $0.1 \mathrm{a}$ \\
$40-40-0-5$ & $922.62 \mathrm{~cd}$ & $0.98 \mathrm{~b}$ & $0.8 \mathrm{a}$ & $21.66 \mathrm{ab}$ & $4.39 \mathrm{~b}$ & $0.05 \mathrm{a}$ \\
$80-40-0-5$ & $1133.93 \mathrm{abcd}$ & $1.14 \mathrm{ab}$ & $0.79 \mathrm{a}$ & $23.09 \mathrm{ab}$ & $5.07 \mathrm{ab}$ & $0.05 \mathrm{a}$ \\
$120-40-0-5$ & $1050.6 \mathrm{bcd}$ & $1.22 \mathrm{ab}$ & $0.8 \mathrm{a}$ & $20.26 \mathrm{~b}$ & $5.02 \mathrm{ab}$ & $0.05 \mathrm{a}$ \\
\hline
\end{tabular}




\begin{tabular}{ccccccc}
\hline $\begin{array}{c}\text { Tratamiento } \\
\text { N-P-K-D }\end{array}$ & $\begin{array}{c}\text { Rendimiento } \\
\left(\mathrm{kg} \mathrm{ha}^{-1}\right)\end{array}$ & $\begin{array}{c}\text { Altura de } \\
\text { planta }(\mathrm{m})\end{array}$ & $\begin{array}{c}\text { Diámetro de } \\
\text { tallo }(\mathrm{cm})\end{array}$ & $\begin{array}{c}\text { Longitud de } \\
\text { panoja }(\mathrm{cm})\end{array}$ & $\begin{array}{c}\text { Ancho de } \\
\text { panoja }(\mathrm{cm})\end{array}$ & IPA \\
\hline $40-40-20-5$ & $1046.13 \mathrm{bcd}$ & $1.33 \mathrm{ab}$ & $0.89 \mathrm{a}$ & $23.67 \mathrm{ab}$ & $6.52 \mathrm{ab}$ & $0.15 \mathrm{a}$ \\
$80-40-20-5$ & $974.7 \mathrm{bcd}$ & $1.21 \mathrm{ab}$ & $0.78 \mathrm{a}$ & $22.33 \mathrm{ab}$ & $5.43 \mathrm{ab}$ & $0.09 \mathrm{a}$ \\
$120-40-20-5$ & $1970.24 \mathrm{a}$ & $1.21 \mathrm{ab}$ & $0.88 \mathrm{a}$ & $22.5 \mathrm{ab}$ & $6.28 \mathrm{ab}$ & $0.07 \mathrm{a}$ \\
\hline
\end{tabular}

$\mathrm{N}=$ nitrógeno; $\mathrm{P}=$ fósforo; $\mathrm{K}=$ potasio $\mathrm{D}=$ densidad de plantas; $\mathrm{IPA}=$ índice de plantas acamadas. Letras diferentes indican medias estadísticamente diferentes (Tukey, 0.05).

Los estudios que se han realizado para determinar cuál es la dosis óptima de nitrógeno en $A$. Hypochondriacus son numerosos; no obstante, los resultados han sido diversos. Por ejemplo, Ojo et al. (2007); Ramírez et al. (2011) reportaron mayores rendimientos de grano con aplicaciones en el intervalo de 60 y $80 \mathrm{~kg} \mathrm{ha}^{-1}$, en tanto que Ansari y Aghaalihkani (2012) obtuvieron mayores rendimientos con dosis de $180 \mathrm{~kg} \mathrm{ha}^{-1}$. Por tanto, se puede decir, que el intervalo de respuesta del cultivo de amaranto a la fertilización nitrogenada es bastante amplio o bien, que encada uno de esos experimentos se utilizaron diferentes razas o variedades de A. Hypochondriacus, cuyos requerimientos nutricionales de este elemento son distintos.

En el caso de la variable rendimiento se encontró respuesta altamente significativa $(p<0.01)$ a los factores $\mathrm{N}, \mathrm{K}$, y las interacciones $\mathrm{N} * \mathrm{~K}$ y $\mathrm{N} * \mathrm{~K} * \mathrm{D}$ (Cuadro 3), esta información es importante a la hora de generar futuras recomendaciones de fertilización debido a que los productores de Tochimilco no realizan fertilización potásica en el cultivo de amaranto (Sánchez et al., 2015). Asimismo, la D no influyó por si solo en el rendimiento de amaranto, lo que coincide con los resultados obtenidos por Kumar y Yassin (2014) pero contrastan con lo reportado por Pourfarid et al. (2014); Olofintoye et al. (2015); Moshaver et al. (2016) quienes reportaron que el factor D por sí mismo si tiene un efecto significativo en el rendimiento.

Cuadro 3. Cuadrados medios de las variables evaluadas en dos ciclos de cultivo.

\begin{tabular}{cccccccc}
\hline Factor & gl & Rendimiento & $\begin{array}{c}\text { Altura de } \\
\text { planta }\end{array}$ & $\begin{array}{c}\text { Diámetro de } \\
\text { tallo }\end{array}$ & $\begin{array}{c}\text { Longitud de } \\
\text { panoja }\end{array}$ & $\begin{array}{c}\text { Ancho de } \\
\text { panoja }\end{array}$ & IPA \\
\hline $\mathrm{REP}$ & 3 & $5764.42 \mathrm{~ns}$ & $0.34^{* *}$ & $0.11^{* *}$ & $275.9^{* *}$ & $17.04^{* *}$ & $0.01 \mathrm{~ns}$ \\
$\mathrm{~N}$ & 2 & $20143.06^{* *}$ & $0.12 \mathrm{~ns}$ & $0 \mathrm{~ns}$ & $6.6 \mathrm{~ns}$ & $1.78 \mathrm{~ns}$ & $0.01 \mathrm{~ns}$ \\
$\mathrm{~K}$ & 1 & $24017.01^{* *}$ & $0 \mathrm{~ns}$ & $0.04 \mathrm{~ns}$ & $25.21 \mathrm{~ns}$ & $3.51 \mathrm{~ns}$ & $0 \mathrm{~ns}$ \\
$\mathrm{D}$ & 2 & $991.43 \mathrm{~ns}$ & $0.11 \mathrm{~ns}$ & $0.11^{* *}$ & $95.65^{* *}$ & $10.98^{* *}$ & $0 \mathrm{~ns}$ \\
$\mathrm{~N} * \mathrm{D}$ & 4 & $6482.45 \mathrm{~ns}$ & $0.02 \mathrm{~ns}$ & $0 \mathrm{~ns}$ & $2.96 \mathrm{~ns}$ & $0.97 \mathrm{~ns}$ & $0.01 \mathrm{~ns}$ \\
$\mathrm{~N} * \mathrm{~K}$ & 2 & $14686.06^{* *}$ & $0.29^{* *}$ & $0.07 \mathrm{~ns}$ & $59.66^{* *}$ & $6.96^{*}$ & $0.02 \mathrm{~ns}$ \\
$\mathrm{~K} * \mathrm{D}$ & 2 & $3942.76 \mathrm{~ns}$ & $0.06 \mathrm{~ns}$ & $0 \mathrm{~ns}$ & $12.81 \mathrm{~ns}$ & $3.55 \mathrm{~ns}$ & $0.01 \mathrm{~ns}$ \\
$\mathrm{~N} * \mathrm{~K} * \mathrm{D}$ & 4 & $31241.12^{* *}$ & $0.07 \mathrm{~ns}$ & $0.04 \mathrm{~ns}$ & $32.1 \mathrm{~ns}$ & $4.26 \mathrm{~ns}$ & $0.02 \mathrm{~ns}$ \\
Error & 51 & & & & & & \\
Total & 71 & & & & & & 22.62 \\
$\mathrm{CV}$ & & 28.13 & 19.18 & 17.43 & 15.09 & 127.28 \\
\hline
\end{tabular}

$\mathrm{gl}=$ grados de libertad; $\mathrm{IPA}=$ índice de plantas acamadas; $\mathrm{REP}=$ repetición; $\mathrm{N}=$ nitrógeno; $\mathrm{K}=$ potasio; $\mathrm{D}=$ densidad de plantas. ${ }^{*}=p<0.05 ;{ }^{* *}=p<0.01 ; \mathrm{ns}=$ no significativo. 
Por lo tanto, el efecto que la D tiene en el rendimiento de amaranto también puede depender de factores como el material genético cultivado, o por el tipo de sistema de producción utilizado, ya sea el caso del sistema convencional de siembra directa en el que hay de 3 a 5 plantas por hoyo de siembra, el sistema convencional de trasplante en el cual, en cada hoyo de siembra se trasplanta solamente una planta por lo que hay menor competencia (Espitia et al., 2010).

Es importante mencionar, que el máximo rendimiento se obtuvo en plantas de porte bajo $(1.21 \mathrm{~m})$, característica que es deseable en plantas de amaranto, debido a que plantas con mayor altura tienen mayor susceptibilidad al acame. Las plantas acamadas que llegan a estar en contacto directo con el suelo son más susceptibles a la enfermedad conocida como el 'carbón del amaranto' la cual es causado por dos especies del hongo Thecaphora: amaranthi y amaranthicola (Bernal et al., 2000).

Respecto a la altura de planta se encontró efecto significativo ( $p<0.01)$ por la interacción $\mathrm{N} * \mathrm{~K}$ y en este caso el factor D no influyó en esta variable (Cuadro 3) situación que contrasta con lo reportado por otros investigadores quienes encontraron efectos individuales significativos de la D en la altura de A. hypochondriacus, aunque de formas diferentes, por ejemplo: Torres et al. (2006); Ramírez et al. (2011) concluyeron que la altura incrementa con una mayor densidad de plantas; en contra parte, Gimplinguer et al. (2007); Romero et al. (2017) determinaron que la altura de planta disminuye con el incremento de la densidad.

En relación con la longitud de panoja, la $\mathrm{D}$ y la interacción $\mathrm{N} * \mathrm{~K}$ influyeron significativamente (cuadro 3). Estos resultados son diferentes a los reportados por Ramírez et al. (2011); Kumar y Yassin (2014) quienes concluyeron que el tamaño de la panoja está en función de las características del material genético utilizado y no de las dosis de fertilización ni la densidad de plantas.

\section{Conclusiones}

La densidad de plantas influye significativamente produciendo plantas de A. Hypochondriacus raza Mercado con mayor diámetro de tallo, longitud y ancho de panoja producido siguiendo el itinerario técnico utilizado en el sistema convencional de siembra directa. Cuando la densidad interacciona con las dosis de fertilización se presentan diferencias significativas en el rendimiento, alcanzando la mayor producción (1 970.24 kg ha ${ }^{-1}$ ) con la aplicación de 120-40-20 kg ha-1 de N-P-K y densidad de 260417 plantas ha ${ }^{-1}$. Por tanto, la decisión que toman los productores de dejar tres, cuatro o cinco plantas por hoyo de siembra al momento de realizar la práctica del aclareo no es algo trivial, sino que es una decisión que impacta significativamente en el rendimiento del cultivo.

\section{Literatura citada}

Alemayehu, F. R.; Bendevis, M. A. and Jacobsen, S. E. 2015. The potential for utilizing the seed crop amaranth (Amaranthus spp.) in East Africa as an alternative crop to support food security and climate change mitigation. J. Agr. Crop Sci. 201(5):321-329. https://doi.org/10.1111/jac.12108.

Ansari, A. S. and Aghaalikhani, M. 2012. Effect of nitrogen and density on qualitative and quantitative performance of forage Amaranth. Iranian J. Agr. Sci. 1(1):35-44.

Bernal, R.; Rodríguez, J.; Estrada, A.; Hernández, A. y Gatica, M. 2000. Microflora asociada a la semilla de amaranto (Amaranthus hypochondriacus L.). Rev. Fitotec. Mex. 23(1):109-118. 
Espitia, E.; Mapes, C.; Escobedo, D.; De la O, M.; Rivas, P.; Martínez, G.; Cortés, L. y Hernández, J. M. 2010. Conservación y uso de los recursos genéticos de amaranto en México. SINAREFI-INIFAP-UNAM. Celaya, Guanajuato, México. 201 p.

Espitia, E.; Escobedo, D.; Núñez, C. A.; Aguilar, M. J.; Rivas, P. y Sesma, L. F. 2020. Confirmación de razas geográficas de amaranto (Amaranthus spp.) por análisis discriminante canónico. Agrociencia. 54(7):927-937. https://doi.org/10.47163/agrociencia. v54i7.2243.

Kumar, S. R. and Yassin, G. M. 2014. Genotypic variability in grain amaranthus (Amaranthus hypochondriacus L.) under varied plant densities. J. Appl. Hortic. 16(2):161-164. https://www.researchgate.net/profile/Ramesh_Selvan/publication/275866632_Genotypic_ variability_in_grain_amaranthus_Amaranthus_hypochondriacus_L_under_varied_plant_ densities/links/554873eb0cf26a7bf4dac284/Genotypic-variability-in-grain-amaranthusAmaranthus-hypochondriacus-L-under-varied-plant-densities.pdf.

Moshaver, E.; Madani, H.; Emam, Y.; Mohamadi, G. N. and Sharifabad, H. H. 2016. Effect of planting date and density on amaranth (Amaranthus hypochondriacus L.) growth indices and forage yield. Journal of Experimental Biol. Agr. Sci. 4(5):541-547. http://www.jebas.org/Jou.Exp.Bio.Agr.Sci/00400531082016/10.18006_2016.

Ojo, O. D.; Kintomo, A. A.; Akinrinde, E. A. and Akoroda, M. O. 2007. Comparative effect of phosphorus sources for grain amaranth production. Comm. Soil Sci. Plant Analysis. 38(12):35-55. https://doi.org/10.1080/00103620601093611.

Olofintoye, J. A. T.; Abayomi, Y. A. and Olugbemi, O. 2015. Yield responses of grain amaranth (Amaranthus cruentus L.) varieties to varying planting density and soil amendment. Afr. J. Agr. Res. 10(21):2218-2225. https://doi.org/10.5897/AJAR2015.9746.

Pourfarid, A.; Kamkar, B. and Akbari, G. A. 2014. The effect of density on yield and some agronomical and physiological traits of Amaranth (Amaranthus spp.). Int. J. Far. Alli. Sci. 3(12):1256-1259. http://ijfas.com/wp-content/uploads/2015/01/1256-1259.pdf.

Ramírez, M.; Espitia, E.; Carballo, A.; Zepeda, R.; Vaquera, H. y Córdoba, L. 2011. Fertilización y densidad de plantas en variedades de amaranto (Amaranthus hypochondriacus L.). Rev. Mex. Cienc. Agríc. 2(6):855-866, 2011. http://www.scielo.org.mx/pdf/remexca/v2n6/ v2n6a5.pdf.

Romero, C. O.; Ocampo, J.; Sandoval, E.; Navarro, H.; Franco, O. y Calderón, F. 2017. Fertilización orgánica-mineral del cultivo de amaranto (Amaranthus hypochondriacus L.). Rev. Mex. Cienc. Agríc. 8(8):1759-1771. https://doi.org/10.29312/remexca.v8i8.700.

Romero, C. O.; Mendoza, J.; Castro, E. y Sánchez, F. 2018. Estrategia para el fortalecimiento de la producción y comercialización de amaranto en Tochimilco, Puebla. Rev. Educateconciencia. 18(19):6-19. https://core.ac.uk/download/pdf/268578914.pdf.

SAS. 2004. Statistical Analysis System Institute inc. The SAS System. Release version 9.0. Institute Inc, Cary, NC. USA. 830 p.

Sánchez, J.; Argumedo, A.; Álvarez, J. F.; Méndez, J. A. y Ortiz, B. 2015. Conocimiento tradicional en prácticas agrícolas en el sistema del cultivo de amaranto en Tochimilco, Puebla. Agr. Soc. Des. 12(2):237-254. http://www.scielo.org.mx/pdf/asd/v12n2/ v12n2a7.pdf.

Sánchez, J.; Argumedo, A.; Álvarez, J. F.; Méndez, J. A. y Ortiz, B. 2016. Análisis económico del sistema sociotécnico del cultivo de amaranto en Tochimilco, Puebla. Acta Universitaria. 26(3):95-104. https://doi: 10.15174/au.2016.888. 
SIAP. Sistema de Información Agroalimentaria y Pesquera. 2017. Cierre de la producción agrícola por estado. https://www.gob.mx/siap.

Torres, S. G.; Trinidad, S. A.; Reyna, T. T.; Castillo, J. H.; Escalante, E. A. y de León González, F. 2006. Respuesta de genotipos de amaranto a densidades de población. Rev. Fitotec. Mex. 29(4):307-312. http://www.redalyc.org/articulo.oa?id=61029405. 